\begin{tabular}{|c|l|}
\hline Title & Surface switching of rotating fluid in a cylinder \\
\hline Author(s) & Suzuki, Toshiyuki; lima, Makoto; Hay ase, Y umino \\
\hline Citation & $\begin{array}{l}\text { Physics of Fluids, 18(10), 101701 } \\
\text { https://doi.org/10.1063/1.2359740 }\end{array}$ \\
\hline Issue Date & 2006-10 \\
\hline Doc URL & http://hdl.handle.net/2115/16007 \\
\hline Rights & Copyright $\odot 2006$ A merican Institute of Physics \\
\hline Type & article \\
\hline File Information & PF18-10.pdf \\
\hline
\end{tabular}

Instructions for use 


\title{
Surface switching of rotating fluid in a cylinder
}

\author{
Toshiyuki Suzuki, Makoto lima, ${ }^{\text {a) }}$ and Yumino Hayase \\ Research Institute for Electronic Science, Hokkaido University, Sapporo 606-0812, Japan
}

(Received 1 June 2006; accepted 8 September 2006; published online 3 October 2006)

\begin{abstract}
We study the surface shape of water in an open cylinder driven by constant rotation of the bottom. Around the critical Reynolds number for the laminar-turbulent transition, the surface deformation, which is of the order of the container size, shows an aperiodic switching phenomenon between an axisymmetric shape and a nonaxisymmetric shape. The axisymmetric shape is observed as a steady state when the Reynolds number is smaller than that in the switching region, while the nonaxisymmetric shape is observed as a (quasi-) periodic state in which the surface rotates at almost constant angular velocity when the Reynolds number is larger than that in the switching region. A detailed analysis for the surface shape suggests that the flow with the nonaxisymmetric shape is turbulent. () 2006 American Institute of Physics. [DOI: 10.1063/1.2359740]
\end{abstract}

Fluid flow with large deformations of the free surface can be seen in daily life: e.g., bathtub vortices, ${ }^{1,2}$ hydraulic jumps, ${ }^{3}$ or water poured into a cup. ${ }^{4}$ The coupling between the free-surface deformation and flow causes difficulties for solving the problem, because, in general, the shape of free surface is dynamically changed due to the flow. In simple cases with steady axisymmetric deformation and laminar flow, detailed analysis has been reported. ${ }^{1,3}$

The axisymmetry of free-surface flow in a cylinder driven by the endwall rotation is broken by an instability when the Reynolds number is about $2200 .^{5-7}$ The Reynolds numbers for the primary and subsequent instabilities are, however, so small that the deformation of the free surface is negligible compared to the radius of the cylinder. Thus the free surface can be approximated as a flat plane on which the slip condition is applied for flow. ${ }^{7-9}$ The flow is laminar in the range of Reynolds numbers, in which small deformations of the free surface are observed.

A laminar-turbulent transition takes place at rather high Reynolds numbers, i.e., roughly $10^{5}$. It is typically observed in the boundary layer near the rotating disk. ${ }^{10-12}$ Because the mean profile of the flow is changed after the transition to turbulence, the shape of the free surface will be changed.

Recently, much attention has been paid for patterns of the surface deformation under turbulent flow. Strong mixing by single or multiple stirrers causes interesting surface patterns including surfaces covered with small polygons, which was found by Tsuji (unpublished). In turbulent TaylorCouette flow, a bistability between a tilted and an untilted free surface has been reported. ${ }^{13}$ Large, polygon-like surface deformations have been observed ${ }^{14}$ in a setup similar to that in Ref. 5.

In this Letter, we experimentally examined the shape and the temporal variation of large surface deformations due to the flow in an open cylinder driven by a rotating bottom. We found several types of surface deformations including axisymmetric and nonaxisymmetric ones. Interestingly, it was

\footnotetext{
a) Telephone: +81-11-706-2412. Fax: +81-11-706-4966. Electronic mail: makoto@nsc.es.hokudai.ac.jp
}

found that the (time-averaged) depth of the deformed surface is not a monotone function of the rotation speed (Reynolds number): the vertical dimension of the surface shows a transition as well as its axial symmetry. Furthermore, we found temporally aperiodic switching of the surface between an axisymmetric and a nonaxisymmetric state. A detailed analysis for the surface shape suggests that the flow with the nonaxisymmetric shape is turbulent.

Figure 1(a) shows a schema of the open cylinder and driving units. Water in the cylinder is driven by a magnetic stirrer (AS-ONE, HPS-500R) via a disk rotor. The disk rotor was made by using a disk-type magnetic stirring bar (AS-ONE), so that the radius of the disk rotor $\left(R^{\prime}=42.0 \mathrm{~mm}\right)$ is tuned to fit the inner radius of the cylinder $(R=42.5 \mathrm{~mm})$. The top of the rotor was covered with glass, the same material as the cylinder, and we regard the top of the rotor as the bottom of the cylinder. The rotation speed is measured by a revolution indicator (Mistral, LHT-04), and the error for the constant rotation was within $1 \%$. The rotor generates rotational flow which causes substantial surface deformation, which was recorded with a digital video camera (SONY, DCR-TRV7) from the side and the top.

The change of the surface shape was characterized by the following two quantities: one is the height of the surface, $h(t)$, defined as the distance between the position of the surface and the bottom on the middle vertical line on a picture from the side [Fig. 1(b-1)]. The other is the axisymmetry of the surface, circular (axisymmetric) or elliptic (nonaxisymmetric). The latter is obtained by the change of the width of the surface at a constant height.

We controlled two parameters. One is the aspect ratio $\mathcal{A} \equiv H / R$ ( $H$ is the unperturbed height). The other is the Reynolds number $\mathcal{R} \equiv 2 \pi \omega R^{2} / \nu$ ( $\omega$ is the frequency of the rotation and $\nu$ is the kinematic viscosity of water, $\nu=1.0$ $\times 10^{-6} \mathrm{~m}^{2} / \mathrm{s}$ at room temperature). The parameters were ranged $0.47<\mathcal{A}<1.18(20 \mathrm{~mm} \leq H \leq 50 \mathrm{~mm})$ and $\mathcal{R}<2.6$ $\times 10^{5}(\omega \leq 23.3 \mathrm{~Hz})$. We mainly report on the result for $A=0.94 \quad(H=40 \mathrm{~mm})$. We increased $\omega$ by steps of $0.07-0.17 \mathrm{~Hz}$. No hysteresis was found when $\omega$ was decreased. 


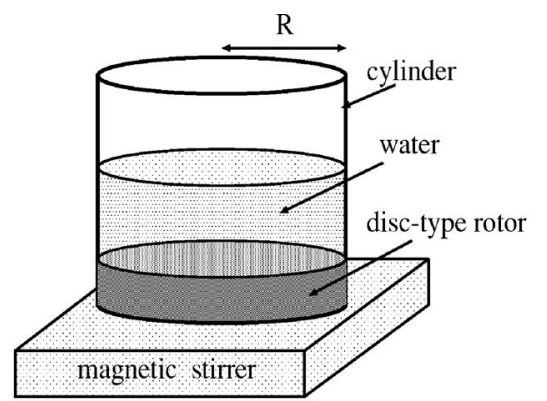

(a)

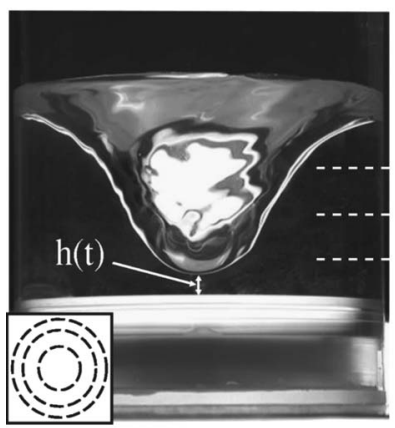

(b-1)

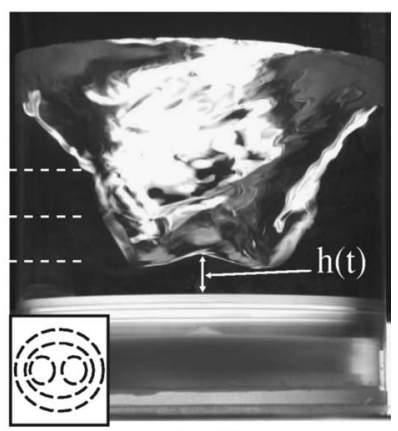

(b-2)
FIG. 1. (a) The schema of the experimental setup. Fluid (tap water) in an open glass cylinder is driven by a disk-type rotor whose radius is close to the inner radius of the cylinder. (b-1) Surface profile for the symmetric state, Sy $\left(\mathcal{A}=0.94, \mathcal{R}=0.823 \times 10^{5} ; \omega=7.25 \mathrm{~Hz}\right)$. The surface has a steady inverted-bell shape. The definition of $h(t)$, the height of the surface at the center, is shown. Inset: Schematic contour plots for the height of the surface. All lines of equal height are circles. (b-2) Surface profile for the asymmetric state, As $\left(\mathcal{A}=0.94, \mathcal{R}=1.65 \times 10^{5} ; \omega=14.6 \mathrm{~Hz}\right)$. The contour plot for this state is elliptic for large heights and has two humps in the center. The surface rotates at almost constant angular velocity.

Figure 2 displays regions of different surface states and the dependence of the average of $h(t),\langle h\rangle$, on the Reynolds number. According to their axial symmetry, the surfaces are classified into four states: symmetric state (Sy), switching state $(\mathrm{Sw})$, asymmetric state (As), and another symmetric state $\left(\mathrm{Sy}^{\prime}\right)$. The experimental error for the Reynolds numbers separating those four states is shown by gray rectangles.

The symmetric state (Sy) is observed when $0<\mathcal{R}$ $<1.31 \times 10^{5}$ : the surface is axisymmetric, i.e., $h(t)$ $=$ constant and horizontal cross sections of the surface are circular [Fig. 1(b-1)]. In this region, $\langle h\rangle$ decreases monotonically with $\mathcal{R}$ and reaches zero at $\mathcal{R}=0.943 \times 10^{5}$. For the range $0.943 \times 10^{5}<\mathcal{R}<1.31 \times 10^{5}$, the surface touches the bottom to make a circular contact line. Correctly, the state which we here call symmetric is already asymmetric as shown by Lopez et al. They proved experimentally and numerically that the surface deformation is asymmetric for small Reynolds numbers $(\mathcal{R}<2500) .{ }^{7}$ The deviations from a symmetric surface are, however, small when compared to the system size. In this sense we label the surface for small Reynolds numbers symmetric.

The asymmetric state (As) is observed in the region $1.58 \times 10^{5}<\mathcal{R}<2.37 \times 10^{5}$. The surface loses the axisymmetry, and two humps develop from the lower part of the surface [Fig. 1(b-2)] and these deviations are of the order of the container. The surface rotates with almost constant period

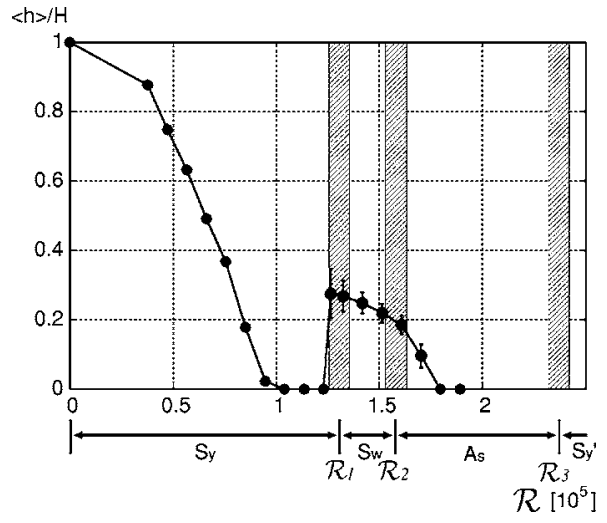

FIG. 2. The phase diagram for the surface shape. A typical measurement of $\langle h\rangle$ is shown as a function of $\mathcal{R}$. The transition Reynolds numbers $\mathcal{R}_{1}, \mathcal{R}_{2}$, and $\mathcal{R}_{3}$ are $1.31 \times 10^{5}, 1.58 \times 10^{5}$, and $2.37 \times 10^{5}$, respectively. The gray regions indicate the standard deviations of the transition points extracted from our measurements. When $\mathcal{R}_{1}<\mathcal{R}<\mathcal{R}_{2}$, the surface aperiodically switches between a state similar to Sy and a state similar to As (switching state, $\mathrm{Sw})$. In the $\mathrm{Sw}$ and As states, $h(t)$ strongly deviates from its mean value, $\langle h\rangle$, as indicated by the error bars.

of $0.35 \mathrm{~s}$ when $\mathcal{R}=1.70 \times 10^{5}$. The contour plot for the height of the surface is elliptic for large heights and has two humps in the center as shown in the inset in Fig. 1(b-2). The error bars shown in Fig. 2 result from a modulation of the surface, i.e., the surface deforms on a time scale $\sim 10 \mathrm{~s}$ in addition to its rotation but stays asymmetric.

A qualitative change in this region is that $\langle h\rangle$ takes positive values again. The surface elongates again with increasing $\mathcal{R}$ and reaches to the bottom at $\mathcal{R}=1.85 \times 10^{5}$. The contact line of the surface with the bottom is then elliptic.

A symmetric state $\left(\mathrm{Sy}^{\prime}\right)$ is observed again if $\mathcal{R}>2.37$ $\times 10^{5}$. The transition from As to $\mathrm{Sy}^{\prime}$ is defined as the Reynolds number for which the contact line to the bottom is circular again. We found a clear transition, ${ }^{15}$ and a detailed analysis will be published elsewhere.

The switching state $(\mathrm{Sw})$ is observed between the Sy region and the As region $\left(1.31 \times 10^{5}<\mathcal{R}<1.58 \times 10^{5}\right)$. Although $\mathcal{R}$ is constant in time, the surface switches aperiodically between two states: an axisymmetric shape $(\widetilde{S y})$ similar to Sy and a rotating nonaxisymmetric shape ( $\widetilde{A s})$ similar to As. The aperiodic switching in the time series of $h(t)$ is shown in Fig. 3(a). There are periods in which $\widetilde{\text { As }}$ [where $h(t)>0]$ is dominant $(P)$, and periods in which $\widetilde{\text { Sy }}$ [where $h(t)=0]$ is dominant $(Q)$. The time scale for the switching from $\widetilde{A s}$ to $\widetilde{\text { Sy }}$ or $\widetilde{\mathrm{Sy}}$ to $\widetilde{\mathrm{As}}$ is about $5 \mathrm{~s}$.

The typical switching loop is shown in Fig. 3(b). The route from $\widetilde{\text { Sy }}$ to $\widetilde{\text { As}}$, which is shown in Fig. 3(b)-(1) to 3(b)-(4) is different from the route from $\widetilde{\text { As }}$ to $\widetilde{\text { Sy which is }}$ shown in Fig. 3(b)-(4) to 3(b)-(8). In the route from $\widetilde{\text { Sy }}$ to $\widetilde{\mathrm{As}}$, the axial symmetry is broken first [Fig. 3(b)-(1)(2)], then the lower part of the surface shape elevates [Fig. 3(b)-(2)(3)(4)]. On the other hand, in the route from $\widetilde{\mathrm{As}}$ to $\widetilde{\mathrm{Sy}}$, the axial symmetry is recovered first [Fig. 3(b)-(5)(6)], then the lower part of the surface elongates to the bottom [Fig. 3(b)-(6)(7)(8)]. The typical loops are, however, imperfect, i.e., not all snapshots in Fig. 3(b)-(1) to Fig. 3(b)-(8) are 


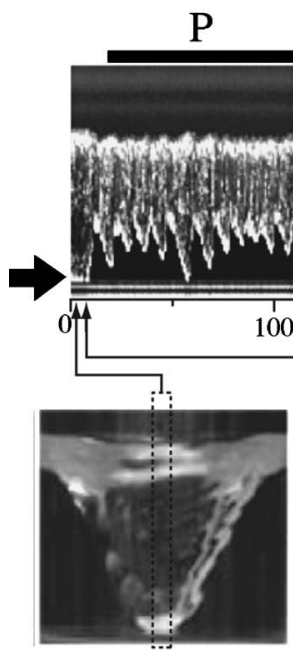

(1)

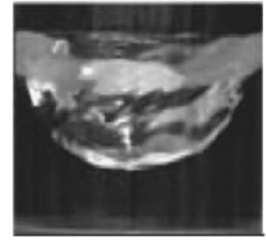

(5)

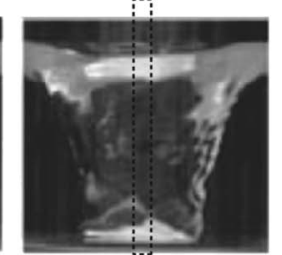

(2)

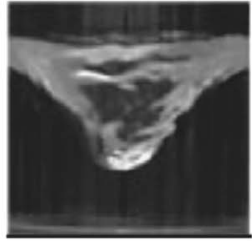

(6)
300

(a)

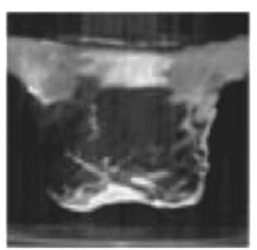

(3)

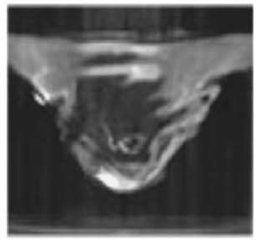

(7)
Q
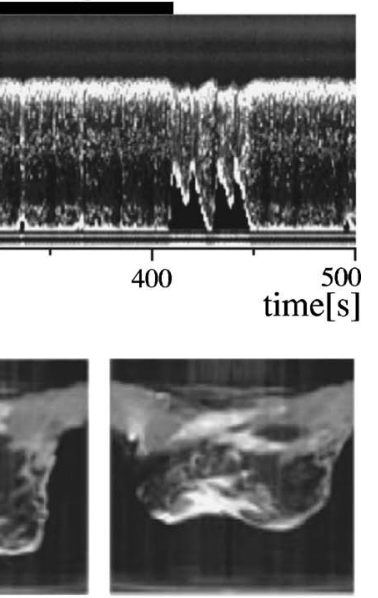

(4)

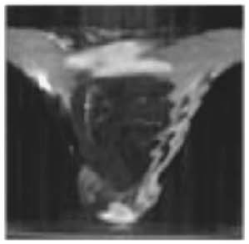

(8)
FIG. 3. Temporal variation of the free surface in the $\mathrm{Sw}$ region at $\mathcal{R}=1.36$ $\times 10^{5} ; \omega=12.0 \mathrm{~Hz}$. (a) Temporal variation of $h$ created by concatenating 5 pixel wide stripes from the center of surface images taken in a time series of $500 \mathrm{~s}$ (one image every $1 \mathrm{~s}$ ). The stripes are schematically indicated in (b)(1) and (b)(2). (b) Snapshots of the surface, which evolve from upper left to lower right. (1)(2)(3)(4): the transition process from the $\widetilde{\text { Sy }}$ state to the $\widetilde{\text { As }}$ state. $(5)(6)(7)(8)$ : the transition process from the $\widetilde{\mathrm{As}}$ state to the $\widetilde{\mathrm{Sy}}$ state.

(b)

observed in a loop. For example, in the sequence shown by $P^{\prime}$, the surface does not touch the bottom [the sequence may consist of Fig. 3(b)-(4)(5)(6)(3)(4)], and in the sequence shown by $Q^{\prime}$, the lower part just elevates in a short time, then elongates to the bottom again [Fig. 3(b)-(1)(2)(3)(7)(1)].

It is suggested that flow characteristics have changed between Sy and As. Figure 2 indicates that we have at least two $\mathcal{R}$ 's that solve the equation $\langle h\rangle=h_{0}\left(h_{0} / H<0.2\right.$ from Fig. 2). The different boundary conditions at the bottom and the volume conservation dictate that mean profiles of the flow as well as the surface should be different for the two $\mathcal{R}$ 's. It is interesting to note that a transition from laminar to turbulent occurs at $\mathcal{R} \sim 10^{5}$, the same order of the critical $\mathcal{R}$ in which the transitions for surface shape and $h(t)$ are observed. Figure 4 shows a power spectrum of $h(t)$ for $\mathcal{R}=1.64 \times 10^{5}$ (As region). The power spectrum of $h(t)$ shows peaks at frequencies $f_{1}$ and $f_{2}$ with supports and their harmonics. The supports become broader as the Reynolds number increases. These suggested that the flow is chaotic, namely turbulent in the As region.

The turbulent flow in the As region might explain the nature of the transition of the surface. If the flow is laminar (ordered), the pressure profile of the fluid on a horizontal cross section takes a minimum at the center. The inhomogeneity of the pressure profile cause the surface to be depressed at the center, which is observed in the Sy region. On the other hand, if the flow is turbulent, the pressure profile is flat in the central region of the cylinder due to the strong mixing. The homogeneity of the pressure profile cause the surface to lift up from the bottom, which is observed in the As region.

The dependency of the transition on the aspect ratio $\mathcal{A}$ is examined. The transition scenario as shown in Fig. 2 is ob- served in the region $\mathcal{A} \geqslant 0.71$, and the transition values of $\mathcal{R}$ increase monotonically with $\mathcal{A}$. For $\mathcal{A}<0.71$, i.e., small amount of water in the cylinder, the region (As, $\langle h\rangle>0$ ) vanishes in the transition scenario, and the $\mathrm{Sw}$ region is sandwiched between the $(\mathrm{Sy},\langle h\rangle=0)$ region and the (As, $\langle h\rangle=0)$ region. ${ }^{15}$ The qualitative change of the transition scenario will be discussed in detail elsewhere.

We note the relevant parameters in this system. Here, the two parameters $\mathcal{A}$ and $\mathcal{R}$ were controlled to organize the surface deformation and the transition. However, an additional dimensionless parameter exists in this system, i.e., the Froude number, in other word, the ratio of the centrifugal

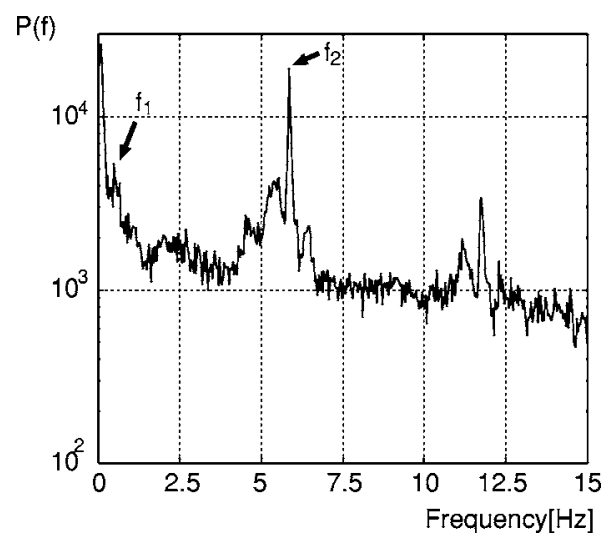

FIG. 4. Power spectrum of $h(t)$ for As $\left(\mathcal{R}=1.64 \times 10^{5} ; \omega=14.5 \mathrm{~Hz}\right)$. The data for $h(t)$ is obtained by image processing for 15000 frames (500 s long). Peaks at $f_{1}=0.492$ and $f_{2}=5.87(\mathrm{~Hz})$ with broad supports (and combinations of them) are recorded. The main frequency $f_{2}$ corresponds to the period of the rotation of the asymmetric shape, $0.35 \mathrm{~s}$, because two humps double the main frequency. 
force to the gravity. To compare our results with other experiments, e.g., Ref. 14, we should arrange the three parameters carefully, which is not achieved in the present study. As a result, we observed only ellipse as a clear polygon pattern. Constructing full phase diagram, including parameter regions of our results and the polygons in Ref. 14, is a most interesting question. However, the construction requires the control of three parameters, thus, we will leave it for future works.

To verify the effect of the boundary conditions at the bottom, we performed further experiment with different rotors. In the case of a stick rotor, we only observed Sy. As and $\mathrm{Sw}$ were not observed. Also, a disk-type rotor with smaller radius $(R=20 \mathrm{~mm}$; height: $17 \mathrm{~mm})$ causes $\mathrm{Sy}, \mathrm{As}$, and $\mathrm{Sy}^{\prime}$, but no distinct $\mathrm{Sw}$ was observed. Thus to observe $\mathrm{Sw}$, we need a disk-type rotor with radius close to that of the cylinder.

We studied the surface deformation caused by the flow in a cylinder driven by the rotation of the bottom. We found an aperiodic switching between an axisymmetric state and a nonaxisymmetric state, in which an asymmetric surface rotates at almost constant angular velocity. It was revealed that the switching process of the surface consists of the change of the symmetry and the elevation and the elongation of the lower part of the surface. The switching state is sandwiched between a stable state with axisymmetric surface for low Reynolds numbers and a stable state with nonaxisymmetric surface for high Reynolds numbers. The switching reported here may be understood as aperiodic transition between these states.

For small Reynolds numbers (small angular velocities of the bottom), the velocity measurement by an ultrasonic velocity profiler (UVP) suggests the rotating wave reported by Hirsa. ${ }^{5}$ The wave is, however, too weak to cause large deformations of the surface. Around $\mathcal{R} \sim 10^{5}$, the symmetry breaking of the surface occurs with large deformation. In the power spectrum of $h(t)$ of the nonaxisymmetric state we found two peaks with broad supports. The result suggests that a strong relation between the interesting surface deformations and the laminar-turbulent transition. The power spectrum of the velocity fluctuation by UVP has also two peaks, each of which reasonably agrees with the power spectrum of $h(t)$. In a forthcoming paper, we will discuss and compare the results of surface shape and flow analysis in terms of a laminar-turbulent transition in detail.

The authors thank Kentaro Ito for taking supplementary data; technical staffs at the Research Institute for Electronic Science, Hokkaido University, for preparing the materials for experiments; and Dr. Kinko Tsuji for leading us to the field of strong-mixing problems. The measurement by UVP was done by Dr. Yuji Tasaka in Hokkaido University.

${ }^{1}$ A. Andersen, T. Bohr, B. Stenum, J. J. Rasmussen, and B. Lautrup, "Anatomy of a bathtub vortex," Phys. Rev. Lett. 91, 104502 (2003).

${ }^{2}$ P. A. Tyvand and K. B. Haugen, "An impulsive bathtub vortex," Phys. Fluids 17, 062105 (2005).

${ }^{3}$ T. Bohr, V. Putkaradze, and S. Watanabe, "Averaging theory for the structure of hydraulic jumps and separation in laminar free-surface flows," Phys. Rev. Lett. 79, 1038 (1997).

${ }^{4}$ H. Kitahata, A. Yamada, and S. Nakata, "Mode bifurcation by pouring water into a cup," J. Chem. Phys. 119, 4811 (2003).

${ }^{5}$ A. H. Hirsa, J. M. Lopez, and R. Miraghaie, "Symmetry breaking to a rotating wave in a lid-driven cylinder with a free surface: Experimental observation," Phys. Fluids 14, L29 (2002).

${ }^{6}$ R. Miraghaie, J. M. Lopez, and A. H. Hirsa, "Flow induced patterning at the air-water interface," Phys. Fluids 15, L45 (2003).

${ }^{7}$ J. M. Lopez, F. Marques, A. H. Hirsa, and R. Miraghaie, "Symmetrybreaking in free-surface cylinder flows," J. Fluid Mech. 502, 99 (2004).

${ }^{8}$ M. Brons, S. K. Voigt, and J. N. Sorensen, "Topology of vortex breakdown bubbles in a cylinder with a rotating bottom and a free surface," J. Fluid Mech. 428, 133 (2001).

${ }^{9} \mathrm{R}$. Iwatsu, "Numerical study of flows in a cylindrical container with rotating bottom and top flat free surface," J. Phys. Soc. Jpn. 74, 333 (2005).

${ }^{10}$ H. L. Reed and W. S. Saric, "Stability of three-dimensional boundary layers," Annu. Rev. Fluid Mech. 21, 235 (1989).

${ }^{11}$ N. Gregory, J. T. Stuart, and W. S. Walker, "On the stability of threedimensional boundary layers with application to the flow due to a rotating disc," Philos. Trans. R. Soc. London, Ser. A 248, 155 (1955).

${ }^{12}$ Y. Kohama, "Study on boundary layer transition of a rotating disk," Acta Mech. 50, 193 (1984).

${ }^{13}$ N. Mujica and D. P. Lathrop, "Bistability and hysteresis in a highly turbulent swirling flow," Physica A 356, 162 (2005).

${ }^{14}$ T. R. N. Jansson, M. P. Haspang, K. H. Jensen, P. Hersen, and T. Bohr, "Polygons on a rotating fluid surface," Phys. Rev. Lett. 96, 174502 (2006).

${ }^{15}$ T. Suzuki, M. Iima, and H. Hayase, "Experimental study on the transitions of fluid flow in a cylinder driven by a rotating disk," RIMS Kokyuroku 1472, 175 (2006) (in Japanese). 